\title{
Melissopalynological and Volatile Compounds Analysis of Buckwheat Honey from Different Geographical Origins and Their Role in Botanical Determination
}

\author{
Sara Panseri, ${ }^{1}$ Alessandra Manzo, ${ }^{2}$ Luca Maria Chiesa, ${ }^{1}$ and Annamaria Giorgi ${ }^{2,3}$ \\ ${ }^{1}$ Department of Veterinary Science and Public Health, Università degli Studi di Milano, Via Celoria 2, 20133 Milan, Italy \\ ${ }^{2}$ Centre for Applied Studies in the Sustainable Management and Protection of the Mountain Environment, Ge.S.Di.Mont., \\ Università degli Studi di Milano, Via Morino 8, 25048 Edolo, Brescia, Italy \\ ${ }^{3}$ Department of Agricultural and Environmental Sciences-Production, Landscape, Agroenergy, \\ Università degli studi di Milano, Via Celoria 2, 20133 Milan, Italy
}

Correspondence should be addressed to Sara Panseri; sara.panseri@unimi.it

Received 4 June 2013; Revised 5 August 2013; Accepted 20 August 2013

Academic Editor: Jose Alberto Pereira

Copyright (c) 2013 Sara Panseri et al. This is an open access article distributed under the Creative Commons Attribution License, which permits unrestricted use, distribution, and reproduction in any medium, provided the original work is properly cited.

\begin{abstract}
Volatile organic compounds (VOCs) have been proposed as one of the main factors for differentiating honeys from different botanical/floral origins. In this work, we investigated the volatile profile of honeys, commercially labeled as buckwheat honeys, from the Alps and its relationship with melissopalynological investigation. The results showed that buckwheat honey samples that contained, to different extents, buckwheat pollen grains on melissopalynological analyses showed similar VOCs profiles, distinguishing them from the other honey floral types analyzed. Among VOCs identified, 3-methylbutanal, butanoic acid, pentanoic acid, and isovaleric acid were considerably greater in the buckwheat honey samples from the Alps. Other compounds were identified only in the honeys containing buckwheat pollen grains such as 3-methyl-2-buten-1-ol, 2-butanone, 2-hydroxy-3-pentanone, 4methylpentanoic acid, 4-pentanoic acid, butanal, 2-methylbutanal, pentanal, dihydro-2-methyl-3(2H)-furanone, 5-methylfurfural, and cis-linalool oxide. These compounds give to buckwheat honey its characteristic aromatic and organoleptic properties and may be considered interesting as potential "variety markers" for botanical determination.
\end{abstract}

\section{Introduction}

Honey is a highly energetic sweet food, produced by bees, used by human beings since ancient times, and is of significant economic value today.

The organoleptic properties of honey, such as flavour, colour, aroma, and texture are essential factors in consumers' estimation of honey quality. These factors are primarily determined by the type of plant species and flowers visited by bees in order to collect nectar or honeydew to produce honey. Climate conditions, bee physiology, honey harvesting and postcollection processing may also influence, to a lesser extent, honey quality. As a consequence, the botanical and, to some extent, also the geographical origins are important characteristics in the evaluation of honey quality [1].
Melissopalynological analyses, consisting of the qualitative and quantitative microscopic examination of honey pollen grains, is, at present, the official test to determine the botanical and geographical origin of honey $[2,3]$. Honey containing pollen mainly collected from a single species is classified as monofloral or unifloral, while multifloral honey contains pollen from lots of different species [4]. However, melissopalynology is time consuming, expensive, and highly influenced by the analyst's subjective ability in interpreting data $[1,5]$. Moreover, the quantity of pollen found in honey is not always directly correlated with the nectar contribution of a species, since, for example, when the honeys are derived from sterile plants, pollen analysis is of absolutely no use [6]. Considering all these limiting factors, efforts in the identification of alternative or complementary methods to replace or 
to integrate pollen analysis are particularly important in order to improve honey quality determination. Studies concerning quality characterization of honey on the basis of phytochemical content in relation with the physicochemical and pollen profile have been published [7-10].

Among phytochemicals, volatile organic compounds (VOCs) have been proposed as one of the main factors for differentiating honeys from different botanical/floral origins [5]. In particular, some unifloral honeys, characterized by individual and specific sensory properties, have been proven to differ one from the other in, among other features, volatile organic composition [11]. Some VOCs are present in the nectar or honeydew collected by bees and could be related to plant characteristics, some others might be originated during honey processing and storage $[12,13]$.

In this paper, the volatile profile of honeys commercially labeled as buckwheat honeys from the Italian Alps, Russia, Nepal, and Poland was analyzed by means of HS-SPME and GC/MS, a valuable method widely used for volatile extraction and analyses $[1,14,15]$. Buckwheat (Fagopyrum esculentum Moench) is an annual, dicotyledonous plant from the Polygonaceae family with a short growing season [16]. It is a multifood-use pseudocereal. Its inflorescence is formed by 7-9 white, pink, or red blossoms [17]. It is a hermaphroditic species which produces self-incompatible flowers pollinated by insects, including bees. The world's largest producer of buckwheat nowadays is China, followed by Russia, Ukraine, and France. In Italy, buckwheat cultivation was introduced around the 16th century [18]. However, during the most recent decades cultivation of this crop has strongly declined and today it survives only in a few alpine valleys $[19,20]$. Buckwheat honey, collected from the little pink flowers by honeybees during the summer, is characterized by a dark purple color, almost black [21]. Buckwheat monofloral honey is mainly produced in North America (Canada and California), China, and in some countries of Europe, such as Poland, Russia, Netherlands, and Germany. Because of the quite low cultivation of buckwheat plants, in Italy, the monofloral buckwheat honey is difficult to produce and it is usually found as a natural component of multifloral honeys [22]. Buckwheat honey is a high-quality product characterized by a sharp, sweet, and slightly biting taste, having beneficial effects on human health due to its antioxidant, bactericidal, and antiinflammatory properties [23-25].

The effectiveness of VOCs markers in the botanical determination of honeys labeled as buckwheat honeys and their relationship with melissopalynological investigation results are discussed.

\section{Materials and Methods}

2.1. Honey Samples. This study was carried out on eight types of honey samples (Table 1). In 2012, 18 samples were bought from local small-scale beekeepers working in Valtellina, an alpine valley of the Lombardy region. In particular, samples B1-B6 (Table 1), labeled as buckwheat honeys, were produced in Teglio (851 $\mathrm{m}$ a.s.l.; min. 352-max. $2.911 \mathrm{~m}$ a.s.1.), a mountain village in Valtellina where buckwheat is still cultivated; M1-M6 were reported as multifloral, A1-A3 as acacia honey
TABLE 1: List of samples. Codes, locations, and honey type reported on the commercial label.

\begin{tabular}{lccc}
\hline $\begin{array}{l}\text { Sample } \\
\text { code }\end{array}$ & Origin & Site & $\begin{array}{c}\text { Honey type on } \\
\text { the label }\end{array}$ \\
\hline B1-B6 & Italy & Valtellina-Lombardy region & Buckwheat \\
B7 & Russia & - & Buckwheat \\
B8-B9 & Poland & - & Buckwheat \\
B10 & Nepal & - & Buckwheat \\
M1-M3 & Italy & Valtellina-Lombardy region & Multifloral \\
M4-M6 & Italy & Valtellina-Lombardy region & Multifloral \\
A1-A3 & Italy & Valtellina-Lombardy region & Acacia \\
R1-R3 & Italy & Valtellina-Lombardy region & Rhododendron \\
\hline
\end{tabular}

(produced in an altitudinal range included between 200 and $1000 \mathrm{~m}$ a.s.l.), and R1-R3 as rhododendron honey (produced over $1000 \mathrm{~m}$ a.s.1.). B7, B8, B9, and B10 honey samples, labeled as buckwheat honeys, from Russia, Poland, and Nepal, respectively, were bought from international traders (Gego Enterpriser Pvt. Ltd., Baneshwor, Kathmandu, Nepal; RATOS-NATURA S.C., Olszownica, 75, 27-552, Backowice, Swietokrzyskie, Poland; Dary Altaya, OOO, Moscow, Russia). The floral origins of buckwheat samples were verified by using melissopalynological analysis. All samples were stored in darkness at a temperature of $4-6^{\circ} \mathrm{C}$ prior to analysis.

2.2. Melissopalynological Analysis. Melissopalynological analysis was performed according to the techniques proposed by the International Commission for Bee Botany (ICBB) and published in 1978 [26]. In this study, all honey samples were analyzed to confirm their floral origin. The microscopic analysis of honey sediment composition provides the percentage of the specific pollen observed by microscopic comparison with known pollen grains (Table 1).

It is necessary to count at least 300 pollen grains for an estimation of the relative frequencies of pollen types and 500 to 1000 pollen grains for the determination of relative frequencies [27]. The examination under the microscope was carried out at the magnification that was most suitable for identifying the various elements in the sediment (400 to $1000 \mathrm{x}$ ). After a first general check to ascertain the main types and densities of pollen grains, the relative frequencies of each pollen type were determined. A count of abortive, irregular, or broken pollen grains, fungal spores, hyphae, and microscopic algae, if they could be identified, was performed.

If the sediment contained a high percentage of overrepresented pollen, a second count excluding the over-represented pollen was done in order to determine more precisely the relative abundance of the other pollen types. The pollen types present in the honey samples were identified, counted, and classified, according to their percentages, as dominant pollen (more than $45 \%$ of the total pollen grains counted), secondary pollen (from 16 to $45 \%$ ), important minor pollen (from 3 to 15\%), and minor pollen (less than 3\%) [28]. 
2.3. HS-SPME Volatile Compounds Sampling from Honey Samples. All the samples were prepared by weighing exactly $5.00 \mathrm{~g}$ of honey in a $20 \mathrm{~mL}$ glass vial, fitted with cap and equipped with silicon/PTFE septa (Supelco, Bellefonte, PA, USA) and by adding $1 \mathrm{~mL}$ of the internal standard solution (IS) in water (1,4-cineol, $1 \mu \mathrm{g} / \mathrm{mL}$, CAS 470-67-7) to check the quality of the fibres. At the end of the sample equilibration period $(1 \mathrm{~h})$, a conditioned $\left(1.5 \mathrm{~h}\right.$ at $\left.280^{\circ} \mathrm{C}\right) 50 / 30 \mu \mathrm{m}$ Divinylbenzene/Carboxen/polydimethylsiloxane (CAR/PDMS/ DVB) StableFlex fibre (Supelco, Bellefonte, PA) was exposed to the headspace of the sample for the extraction $(180 \mathrm{~min})$ by CombiPAL system injector autosampler (CTC analytics, Switzerland). The fibre and the time of extraction used in this study were selected after preliminary study, and the data were reported in Figure 1. The best adsorption of analyte was obtained using CAR/PDMS/DVB and $180 \mathrm{~min}$ as extraction time. The extraction temperature of $25^{\circ} \mathrm{C}$ was selected in order to prevent possible matrix alterations (oxidation of some compounds, particularly aldehydes and furans).

To keep a constant temperature during analysis, the vials were maintained on a heater plate (CTC Analytics, Zwingen, Switzerland). As demonstrated in other researches in which the VOCs profile of food is investigated, the use of high extraction temperature can lead to ex novo formation of volatile compounds or to the production of artefacts $[29,30]$.

2.4. Gas Chromatography-Mass Spectrometry Analysis of VOCs. HS-SPME analysis was performed using a Trace GC Ultra (Thermo-Fisher Scientific, Waltham, MA, USA) Gas Chromatograph coupled to a quadrupole Mass Spectrometer Trace DSQ (Thermo-Fisher Scientific, Waltham, MA, USA) and equipped with an Rtx-Wax column (30 m; $0.25 \mathrm{~mm}$ i.d.; $0.25 \mu \mathrm{m}$ film thickness, Restek, USA). The oven temperature program was: from $35^{\circ} \mathrm{C}$, hold $8 \mathrm{~min}$, to $60^{\circ} \mathrm{C}$ at $4^{\circ} \mathrm{C} / \mathrm{min}$, then from $60^{\circ} \mathrm{C}$ to $160^{\circ} \mathrm{C}$ at $6^{\circ} \mathrm{C} / \mathrm{min}$, and finally from $160^{\circ} \mathrm{C}$ to $200^{\circ} \mathrm{C}$ at $20^{\circ} \mathrm{C} / \mathrm{min}$. Carryover and peaks originating from the fibre were regularly assessed by running blank samples. After each analysis, fibres were immediately thermally desorbed in the GC injector for $5 \mathrm{~min}$ at $250^{\circ} \mathrm{C}$ to prevent contamination. The injections were performed in splitless mode $(5 \mathrm{~min})$. The carrier gas was helium at a constant flow of $1 \mathrm{~mL}^{-1}$. The transfer line to the mass spectrometer was maintained at $230^{\circ} \mathrm{C}$, and the ion source temperature was set at $250^{\circ} \mathrm{C}$. The mass spectra were obtained by using a mass selective detector with the electronic impact at $70 \mathrm{eV}$, a multiplier voltage of $1456 \mathrm{~V}$, and by collecting the data at rate of $1 \mathrm{scan} \mathrm{s}^{-1}$ over the $m / z$ range of $30-350$. Compounds were identified by comparing the retention times of the chromatographic peaks with those of authentic compounds analyzed under the same conditions when available. The identification of MS fragmentation patterns was performed either by comparison with those of pure compounds or using the National Institute of Standards and Technology (NIST) MS spectral database. Volatile compounds measurements from each headspace of honey extracts were carried out by peak area normalization (expressed in percentage). All analyses were done in duplicate.

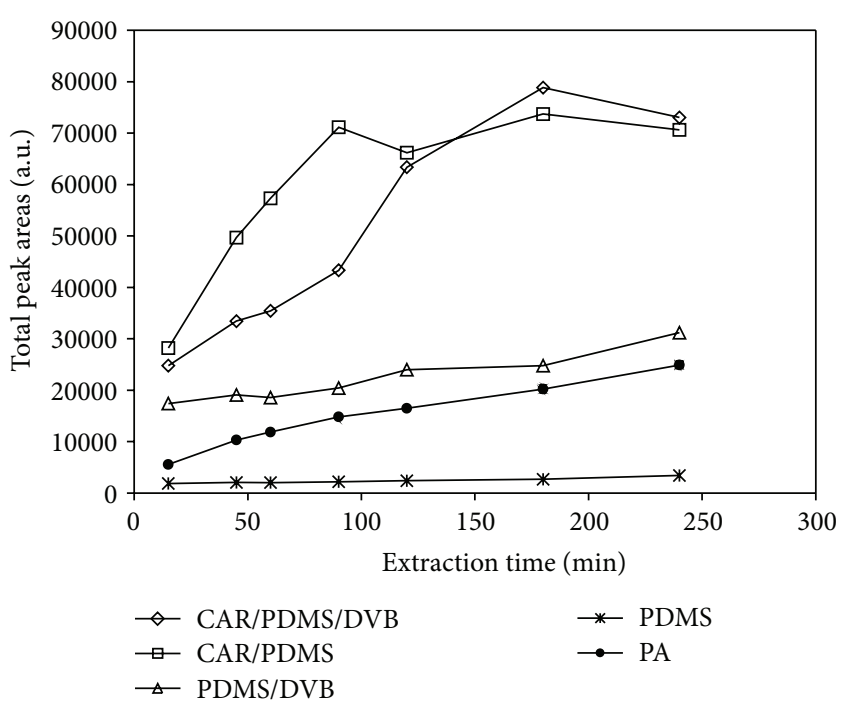

FIgURE 1: Total absorption peak areas (arbitrary unit) for CAR/ PDMS/DVB, CAR/PDMS, PDMS/DVB, PDMS, and PA fibres at different extraction time $(15,45,60,90,120,180$, and $240 \mathrm{~min})$.

\section{Results and Discussion}

3.1. Melissopalynological Analysis. The results of the melissopalynological analyses of the honeys labeled as buckwheat honeys are reported in Tables 2 and 3. The results showed that the buckwheat honey samples B1, B2, and B3 could be classified as monofloral with $45.5 \%, 52 \%$, and $46 \%$ of buckwheat pollen. In Valtellina, the maximum altitude at which buckwheat is cultivated is $1200 \mathrm{~m}$ a.s.l., and its flowering period is August, thus, giving an indication on the elevation and the period of honey samples $\mathrm{B} 1, \mathrm{~B} 2$, and $\mathrm{B} 3$ production.

On the contrary, honey samples B4, B5, and B6, also labeled as buckwheat honeys, had to be classified as multifloral, with a very low $(5 \%, 4.5 \%$, and $5.6 \%$, resp.) relative frequency of buckwheat pollen. The high presence of pollen grains of plants belonging to the Rhododendron genus, growing at an elevation between 1600 and $2300 \mathrm{~m}$ a.s.l. and flowering from June to mid-July, suggested that honey samples B4 and $\mathrm{B} 5$ were produced in Valtellina at higher altitude and in a different season compared to honey samples B1, B2, and B3. The high presence of pollen grains of Eryngium alpinum L. and Euphrasia officinalis L. in honey sample B6 also suggested that it was produced at higher altitude compared to honey samples B1, B2, and B3.

Finally, the presence of pollen grains of plants belonging to the Clematis genus, flowering from May to July, and Lotus alpinus (DC.) Schleicher, growing from 1700 to $2700 \mathrm{~m}$ a.s.l. and flowering in July, seemed to confirm the different period and environment of production of honey samples B4, B5, and B6.

The Polish (B8 and B9) and Nepali (B10) samples had to be classified as multifloral honeys with a prevalence of buckwheat pollen grain $(25 \%, 30 \%$, and $16 \%$, resp.), while the Russian sample (B7) had to be classified as multifloral with $5 \%$ of buckwheat honey. The presence of pollen grains from 
TABLE 2: Relative frequencies of the main pollen types in honeys labeled as buckwheat honeys. B1, B2, and B3: buckwheat honey from Italy (Valtellina); B4, B5, and B6: buckwheat honey from Italy (Teglio).

\begin{tabular}{|c|c|c|c|c|c|c|}
\hline & \multicolumn{6}{|c|}{ Sample code } \\
\hline & B1 & B2 & B3 & B4 & B5 & B6 \\
\hline $\begin{array}{l}\text { Dominant } \\
\text { pollen } \\
(>45 \%) \\
\end{array}$ & F. esculentum $45.5 \%$ & F. esculentum $52 \%$ & $\begin{array}{c}\text { F. esculentum } \\
46 \%\end{array}$ & - & Melilotus 79\% & \\
\hline $\begin{array}{l}\text { Secondary } \\
\text { pollen } \\
(16-45 \%)\end{array}$ & - & - & Robinia 16.5\% & $\begin{array}{l}\text { Rhododendron } 36 \% \\
\text { Clematis } 7.2 \%\end{array}$ & $\begin{array}{l}\text { Rhododendron } \\
21 \%\end{array}$ & $\begin{array}{c}\text { Euphrasia } \\
\text { officinalis 37\% } \\
\text { Compositae } 18 \% \\
\text { Eryngium } \\
\text { alpinum } 17.8 \%\end{array}$ \\
\hline $\begin{array}{l}\text { Important } \\
\text { minor pollen } \\
(3-15 \%)\end{array}$ & $\begin{array}{c}\text { Trifolium repens } \\
10.3 \% \\
\text { Robinia } 8.5 \% \\
\text { Tilia } 3 \%\end{array}$ & $\begin{array}{c}\text { Trifolium repens } 8.7 \% \\
\text { Hedera } 5.2 \%\end{array}$ & $\begin{array}{c}\text { Salix } 8 \% \\
\text { Rubus } 4.7 \%\end{array}$ & $\begin{array}{c}\text { Clematis } 8.3 \% \\
\text { F. esculentum 5\% } \\
\text { Lotus alpinus 5.4\% }\end{array}$ & $\begin{array}{c}\text { F. esculentum } \\
4.5 \% \\
\text { Rubus } 4 \% \\
\text { Mentha } 4 \% \\
\text { Eryngium } \\
\text { alpinum } 4 \% \\
\end{array}$ & $\begin{array}{c}\text { F. esculentum } \\
5.6 \% \\
\text { Mentha } 8 \% \\
\text { Clematis } 5 \% \\
\text { Rubus } 4.3 \%\end{array}$ \\
\hline $\begin{array}{l}\text { Minor pollen } \\
(<3 \%)\end{array}$ & $\begin{array}{c}\text { Sedum/Sempervivum } \\
2 \% \\
\text { Prunus } 2.8 \% \\
\text { Gleditsia } 2.2 \% \\
\text { Pyrus/Malus } 1.9 \% \\
\text { Acer } 1.5 \% \\
\text { Salix } 0.7 \% \\
\text { Clematis } 0.4 \% \\
\text { Trifolium pratense } \\
<1 \% \\
\text { Umbelliferae }<1 \%\end{array}$ & $\begin{array}{c}\text { Verbena } 2 \% \\
\text { Castanea } 1.8 \% \\
\text { Pyrus/Malus } 1 \% \\
\text { Salix } 0.4 \% \\
\text { Sedum } / \text { Sempervivum } \\
<1 \% \\
\text { Knautia/Scabiosa }<1 \%\end{array}$ & $\begin{array}{c}\text { Tilia } 2 \% \\
\text { Ericaceae } 2 \% \\
\text { Compositae } 1.8 \% \\
\text { Trifolium repens } \\
1.5 \% \\
\text { Umbelliferae }<1 \% \\
\text { Achillea spp. }<1 \%\end{array}$ & $\begin{array}{c}\text { Trifolium repens 3\% } \\
\text { Prunus } 2.6 \% \\
\text { Ranunculaceae } 1.7 \% \\
\text { Robinia } 1 \% \\
\text { Campanulaceae } 0.9 \% \\
\text { Trifolium pretense } \\
0.7 \% \\
\text { Acer } 0.7 \% \\
\text { Ranunculaceae } 0.7 \% \\
\text { Umbelliferae } 0.4 \% \\
\text { Salvia }<1 \% \\
\text { Sedum/Sempervivum } \\
<1 \%\end{array}$ & $\begin{array}{c}\text { Compositae } 2 \% \\
\text { Lotus alpinus } \\
2 \% \\
\text { Prunus } 1.8 \% \\
\text { Compositae } 1 \% \\
\text { Trifolium } \\
\text { repens }<1 \% \\
\text { Salix }<1 \%\end{array}$ & $\begin{array}{c}\text { Prunus } 2 \% \\
\text { Umbelliferae } 1.8 \% \\
\text { Hedera } 0.9 \% \\
\text { Salvia } 0.2 \%\end{array}$ \\
\hline
\end{tabular}

TABLE 3: Relative frequencies of the main pollen types in honeys labeled as buckwheat honeys. B7: buckwheat honey from Russia; B8 and B9: buckwheat honey from Poland; B10: buckwheat honey from Nepal.

\begin{tabular}{|c|c|c|c|c|}
\hline & \multicolumn{4}{|c|}{ Sample code } \\
\hline & B7 & B8 & B9 & $\mathrm{B} 10$ \\
\hline $\begin{array}{l}\text { Dominant pollen } \\
(>45 \%)\end{array}$ & - & - & - & Cruciferae $67 \%$ \\
\hline $\begin{array}{l}\text { Secondary pollen } \\
(16-45 \%)\end{array}$ & $\begin{array}{c}\text { Helianthus } 30 \% \\
\text { Melilotus } 24 \%\end{array}$ & Fagopyrum esculentum $25 \%$ & $\begin{array}{c}\text { Fagopyrum esculentum } 30 \% \\
\text { Brassica napus } 17.9 \%\end{array}$ & $\begin{array}{c}\text { Fagopyrum } \\
\text { esculentum } 16 \%\end{array}$ \\
\hline $\begin{array}{l}\text { Important minor } \\
\text { pollen } \\
(3-15 \%)\end{array}$ & $\begin{array}{c}\text { Cruciferae 9\% } \\
\text { Fagopyrum } \\
\text { esculentum 5\% } \\
\text { Trifolium repens 5\% } \\
\text { Echium 4\% } \\
\text { Verbascum 4\% } \\
\text { Robinia 3\% } \\
\end{array}$ & $\begin{array}{c}\text { Brassica napus } 14 \% \\
\text { Echium } 8 \% \\
\text { Trifolium repens } 4 \% \\
\text { Helianthus 3\% } \\
\text { Umbelliferae 3\% }\end{array}$ & $\begin{array}{c}\text { Echium } 13 \% \\
\text { Gleditsia } 12.4 \% \\
\text { Helianthus } 4.8 \%\end{array}$ & $\begin{array}{c}\text { Helianthus } 4 \% \\
\text { Compositae } 4 \% \\
\text { Eucalyptus } 3 \%\end{array}$ \\
\hline $\begin{array}{l}\text { Minor pollen } \\
(<3 \%)\end{array}$ & $\begin{array}{c}\text { Labiatae } 2 \% \\
\text { Onobrychis } 2 \% \\
\text { Cynoglossum } 1 \% \\
\text { Prunus } 1 \% \\
\text { Campanulaceae } 1 \% \\
\text { Compositae } 1 \% \\
\text { Lotus } 1 \% \\
\text { Umbelliferae } 1 \%\end{array}$ & $\begin{array}{c}\text { Lotus } 2 \% \\
\text { Trifolium repens } 1 \% \\
\text { Compositae }<1 \% \\
\text { Sedum } / \text { Sempervivum }<1 \%\end{array}$ & $\begin{array}{c}\text { Sedum/Sempervivum } 3 \% \\
\text { Lamium } 2.3 \% \\
\text { Trifolium repens } 1.9 \% \\
\text { Salix } 1.5 \% \\
\text { Lotus corniculatus } 1.5 \% \\
\text { Umbelliferae } 1.5 \% \\
\text { Salvia } 1.5 \% \\
\text { Euphorbiaceae }<1 \%\end{array}$ & $\begin{array}{c}\text { Rosaceae } 2 \% \\
\text { Umbelliferae } 2 \% \\
\text { Bombacaceae } 1 \% \\
\text { Castanea } 1 \% \\
\text { Labiatae }<1 \%\end{array}$ \\
\hline
\end{tabular}


plants of the Bombacaceae genus in the Nepali honey sample confirmed its Asiatic origin, suggesting it was produced in a tropical or subtropical area of Nepal, where plants of this genus grow. Finally, as Poland is one of the major producers of canola in Europe, the presence of a relevant quantity of Brassica napus L. pollen grains in honey samples B9 and B10 was coherent with its declared origin.

The results regarding the other honey samples (M1-M6, $\mathrm{A} 1-\mathrm{A} 3$, and R1-R3) confirmed the botanical classification reported on the commercial label (data not shown), no buckwheat pollen grain were recovered.

3.2. Analysis of VOCs in Honey Samples. Honey volatiles are a very complex mixture of substances frequently occurring at a very low concentration and with poor chemical stability. Thus, as reported by many authors [2, 31-33], the use of headspace solid-phase-microextraction (HS-SPME) and gaschromatography/mass-spectrometry (GC/MS), a very sensible and solvent-free method for extraction and analyses of this chemical fraction, is particularly suitable. In our experimental conditions, 86 compounds have overall been identified in the honey samples analyzed, and they are summarized in Tables 4 and 5.

These compounds belonged to different major chemical classes as follows: alcohols, phenols, ketones, free fatty acid, esters, aldehydes, furans, and terpenes.

This paper is the first investigation on the VOCs profile of a buckwheat monofloral honey from Valtellina (North of Italy). Remarkable differences in VOCs profiles were observed when comparing honey samples of different floral origins. Consistent with other authors [34-36], most of the compounds were identified in all of the analyzed honeys, but the proportion in which they occurred appeared very different taking into account the different floral/botanical origin. Similarly, in each of the analyzed samples there were compounds which were not present in other types of honeys to be evaluated as potential "floral markers".

The VOCs profile of honeys labeled as buckwheat honeys (B1-B10) was similar, particularly for samples containing a relevant quantity of buckwheat pollen grains despite the different geographical origin. In addition, comparing it with the VOCs profile of the other honey samples, not containing buckwheat pollen grains (M1-M6, A1-A3, and R1-R3), some differences were identified, particularly regarding the composition and the concentration of some chemical classes such as alcohols, aldehydes, free fatty acids, furans, and terpenes as shown in Tables 4 and 5.

Buckwheat honey is characterized by a sharp, sweet, and slightly biting taste, and its organoleptic characteristics have been proven to be reflected in its composition and concentrations of volatile compounds [33]. Moreover, among the aldehydes, methylbutanals have been reported to be responsible for the characteristic pungent, sweetish, and malty flavour of buckwheat honey [22, 37].

In our experimental condition, 3-methylbutanal was found in highest concentration in buckwheat honey, and 2methylbutanal was found to be present only in the honeys containing buckwheat pollen grains. These compounds are commonly found in barley malt [38]. They are known to be Strecker aldehydes, and their presence in honeys is usually associated with the Maillard browning reactions.

The extremely high amounts of methylbutanals in buckwheat honeys compared with some other honeys suggested that this type of honey contains a higher abundance of Strecker degradation precursors, such as amino acids, which would result in a honey with an aroma resembling that which develops upon heat-promoted chemical reactions that occur during the malting of barley [22]. The presence of other Maillard reaction products such as phenylacetaldehyde and dimethyl sulfide supports this hypothesis.

As reported in the literature [33], besides aldehydes, also the concentrations of free fatty acids, like butanoic acid and pentanoic acid, were considerably greater in the honey samples B1, B2, B3, and B9, those containing the higher quantity of buckwheat pollen grains. Butanoic acid gives buckwheat honey its typical pungent smell and pentanoic acid has a rancid smell and an acid taste [37]. Such chemical compounds, characterized by high concentrations in a honey type and specific sensory properties, are to be considered interesting as potential "variety markers" [33].

Wolski et al. [32] reported butanal, phenol, trans-linalool oxide, 3,4,5-trimethylphenol as buckwheat honey marker compounds because they were not found in other honey types. In this study, only butanal was confirmed to be such a marker, being present only in the two honey samples containing a relevant quantity of buckwheat pollen grains, corresponding to the Italian monofloral buckwheat honey (samples B1, B2, and B3) and the Polish honeys (samples B8 and B9).

In the same honey samples, we also found significantly great quantities of isovaleric acid, never reported before in buckwheat honey. Isovaleric acid is a potent, odorant, volatile compound, exhibiting an unpleasant odor associated with the rank smell of perspiring feet and has been considered to be an important off-flavor compound in honeys [39]. Isovaleric acid, has been found to be an important odorant for Anarcardium occidentale L. and Croton sp. honeys from Brazil [40].

Finally, we have identified additional characteristic volatile compounds of buckwheat honey such as 3-methyl-2buten-1-ol, 2-butanone, 2-hydroxy-3-pentanone, 4-methylpentanoic acid, 4-pentanoic acid, butanal, 2-methylbutanal, pentanal, dihydro-2-methyl-3(2H)-furanone, 5-methylfurfural, and cis-linalool oxide. Pasini et al. [21] reported 5methylfurfural and other furans as important buckwheat honey marker compounds. In the literature, furanic compounds were reported to derive from sugar degradation and considered to be indicators of thermal processes and storage [41].

\section{Conclusion}

Honey samples labeled as buckwheat honey, found to contain, to different extents, buckwheat pollen grains on melissopalynological analyses, show similar VOCs profiles, distinguishing them from the other honey floral types analyzed. In particular, the honey samples from the Italian Alps, classified as monofloral buckwheat honey, and the two samples from 
TABLE 4: Volatile compounds identified in investigated honey samples expressed as percentages. B1-B6: buckwheat honey from Italy (TeglioValtellina); B7: buckwheat honey from Russia; B8 and B9: buckwheat honey from Poland; B10: Buckwheat honey from Russia.

\begin{tabular}{|c|c|c|c|c|c|c|c|c|c|c|c|c|}
\hline \multirow{2}{*}{ Compounds } & \multirow{2}{*}{$\mathrm{RT}^{\mathrm{a}}$} & \multirow{2}{*}{ Identification $^{\mathrm{b}}$} & \multicolumn{10}{|c|}{ Sample code } \\
\hline & & & $B 1^{\mathrm{c}}$ & $\mathrm{B} 2^{\mathrm{c}}$ & $B 3^{\mathrm{c}}$ & $\mathrm{B} 4^{\mathrm{c}}$ & $\mathrm{B} 5^{\mathrm{c}}$ & $B 6^{\mathrm{c}}$ & $B 7^{\mathrm{d}}$ & $B 8^{\mathrm{d}}$ & $B 9^{d}$ & $\mathrm{~B} 10^{\mathrm{d}}$ \\
\hline \multicolumn{13}{|l|}{ Alcohols } \\
\hline Ethanol & 3.97 & MS, LRI & 14.85 & 9.05 & 12.25 & 5.70 & 5.18 & 7.65 & 8.39 & 11.41 & 12.00 & 6.36 \\
\hline 2,3-Butanedione & 4.98 & MS, LRI & 0.07 & 0.09 & 0.06 & 0.07 & 0.07 & 0.09 & 0.42 & 0.13 & 0.11 & 2.78 \\
\hline 2-Methyl-3-buten-2-ol & 7.39 & MS, LRI & 0.03 & 0.04 & 0.02 & 0.35 & 0.42 & 0.48 & 0.27 & nd & 0.05 & nd \\
\hline 1-Butanol & 12.67 & MS, LRI & 0.36 & 0.49 & 0.30 & 0.09 & 0.11 & 0.13 & 0.08 & 0.17 & 0.19 & 0.06 \\
\hline 2-Methylbutanol & 14.46 & MS, LRI & 0.01 & 0.01 & 0.01 & 0.05 & 0.06 & 0.03 & 0.02 & 0.03 & 0.03 & 0.08 \\
\hline 2-Methylpropanol & 15.02 & MS, LRI & 0.01 & 0.02 & 0.01 & nd & nd & 0.01 & 0.02 & nd & nd & nd \\
\hline 3-Methylbutanol & 15.41 & MS, LRI & 0.87 & 1.17 & 0.72 & 0.95 & 1.12 & 1.29 & 1.03 & 1.00 & 0.92 & 0.90 \\
\hline 2-Methyl-2-buten-1-ol & 16.78 & MS, LRI & 0.07 & 0.09 & 0.05 & 0.23 & 0.28 & 0.32 & 0.42 & nd & 0.03 & 0.17 \\
\hline 3-Methyl-2-buten-1-ol & 16.94 & MS, LRI & 0.02 & 0.02 & 0.02 & 0.03 & 0.03 & 0.04 & 0.05 & 0.04 & 0.02 & 0.01 \\
\hline 2-Methylbutenol & 19.09 & MS, LRI & 0.01 & 0.01 & 0.01 & 0.03 & 0.03 & 0.03 & nd & nd & 0.03 & nd \\
\hline Total & & & 16.30 & 11.00 & 13.45 & 7.50 & 7.29 & 10.07 & 10.68 & 12.95 & 13.38 & 10.36 \\
\hline \multicolumn{13}{|l|}{ Phenols } \\
\hline 2-Caren-10-al & 29.28 & MS, LRI & 0.01 & 0.01 & 0.01 & 0.03 & 0.06 & 0.07 & 0.05 & nd & nd & 0.14 \\
\hline p-Cymen-8-ol & 30.46 & MS, LRI & 0.03 & 0.04 & 0.03 & 0.01 & 0.01 & 0.01 & 0.05 & 0.03 & 0.05 & 0.07 \\
\hline Phenol & 32.53 & MS, LRI & 0.03 & 0.04 & 0.02 & 0.01 & 0.01 & 0.02 & 0.05 & 0.06 & 0.04 & nd \\
\hline p-Cresol & 33.26 & MS, LRI & 0.19 & 0.26 & 0.16 & 0.01 & 0.02 & 0.03 & 0.20 & 0.01 & 0.09 & nd \\
\hline Thymol & 34.34 & MS, LRI & 0.01 & 0.01 & nd & nd & nd & nd & nd & nd & nd & nd \\
\hline Total & & & 0.27 & 0.36 & 0.21 & 0.06 & 0.11 & 0.12 & 0.35 & 0.11 & 0.18 & 0.21 \\
\hline \multicolumn{13}{|l|}{ Ketones } \\
\hline 2-Butanone & 6.61 & MS, LRI & nd & nd & nd & 0.33 & 0.78 & 0.70 & 0.35 & nd & nd & 0.08 \\
\hline 3-Hydroxy-2-butanone & 17.65 & MS, LRI & 1.06 & 1.44 & 0.88 & 0.44 & 0.52 & 0.60 & 1.09 & 0.80 & 1.23 & 1.26 \\
\hline Hydroxyacetone & 18.08 & MS, LRI & 2.36 & 3.18 & 1.95 & 1.50 & 1.76 & 2.02 & 2.15 & 1.30 & 2.23 & 1.40 \\
\hline 2-Hydroxy-3-pentanone & 19.90 & MS, LRI & 0.02 & 0.03 & 0.02 & 0.03 & 0.04 & 0.04 & 0.04 & 0.04 & 0.02 & 0.03 \\
\hline 1-Hydroxy-2-butanone & 20.29 & MS, LRI & 0.09 & 0.12 & 0.07 & 0.32 & 0.38 & 0.44 & 0.24 & 0.02 & 0.15 & 0.02 \\
\hline 2-Methyl-butyrolactone & 25.20 & MS, LRI & 0.45 & 0.61 & 0.37 & 0.09 & 0.11 & 0.12 & 1.05 & 0.18 & 0.08 & nd \\
\hline 4-Oxoisophorone & 27.42 & MS, LRI & 0.06 & 0.04 & 0.05 & 0.05 & 0.06 & 0.07 & 0.28 & 0.13 & 0.08 & 0.03 \\
\hline Total & & & 4.52 & 6.06 & 3.73 & 3.45 & 4.45 & 4.94 & 6.02 & 2.62 & 8.42 & 3.50 \\
\hline \multicolumn{13}{|l|}{ Free fatty acid } \\
\hline Acetic acid & 22.14 & MS, LRI & 9.35 & 12.63 & 7.72 & 11.28 & 13.25 & 15.22 & 20.14 & 26.80 & 20.48 & 31.80 \\
\hline Formic acid & 23.36 & MS, LRI & 6.65 & 8.98 & 5.49 & nd & 13.25 & 0.14 & nd & 6.29 & 5.90 & 4.41 \\
\hline Propanoic acid & 24.28 & MS, LRI & 0.39 & 0.52 & 0.342 & 0.30 & 0.36 & 0.41 & 1.04 & 0.42 & 0.39 & 0.84 \\
\hline Isobutyric acid & 24.95 & MS, LRI & 0.49 & 0.67 & 0.41 & 0.24 & 0.28 & 0.32 & 0.35 & 0.50 & 0.52 & 2.08 \\
\hline Butanoic acid & 26.19 & MS, LRI & 2.70 & 3.64 & 2.22 & 0.63 & 0.74 & 0.85 & 1.99 & 1.01 & 3.01 & 1.55 \\
\hline 2-Propanoic acid & 26.28 & MS, LRI & nd & nd & nd & nd & nd & nd & nd & nd & nd & nd \\
\hline Isovaleric acid & 27.05 & MS, LRI & 10.49 & 14.17 & 8.66 & 3.02 & 3.55 & 4.08 & 3.35 & 7.00 & 11.26 & 3.96 \\
\hline Pentanoic acid & 28.35 & MS, LRI & 1.46 & 1.98 & 1.21 & 0.15 & 0.18 & 0.20 & 1.51 & 0.18 & 1.62 & 0.66 \\
\hline 2-Butenoic acid & 28.95 & MS, LRI & 0.02 & 0.03 & 0.02 & 0.02 & 0.01 & 0.01 & 0.22 & 0.01 & 0.01 & 0.11 \\
\hline 3-Methylpentanoic acid & 29.40 & MS, LRI & 0.08 & 0.11 & 0.07 & 0.05 & 0.06 & 0.07 & 0.11 & 0.08 & 0.05 & 0.07 \\
\hline 4-Methylpentanoic acid & 29.58 & MS, LRI & 0.04 & 0.05 & 0.03 & 0.02 & 0.02 & 0.03 & 0.41 & 0.03 & 0.03 & 0.01 \\
\hline 4-Pentanoic acid & 30.84 & MS, LRI & 0.02 & 0.03 & 0.02 & 0.01 & 0.01 & 0.03 & 0.05 & 0.03 & 0.02 & 0.01 \\
\hline Benzyl nitrile & 31.63 & MS, LRI & 0.01 & 0.01 & 0.01 & 0.01 & 0.01 & 0.01 & 0.01 & nd & 0.01 & nd \\
\hline Heptanoic acid & 31.96 & MS, LRI & 0.01 & 0.02 & 0.01 & 0.03 & 0.03 & 0.04 & 0.02 & 0.07 & 0.02 & 0.12 \\
\hline Octanoic acid & 33.05 & MS, LRI & 0.02 & 0.02 & 0.01 & 0.03 & 0.03 & 0.03 & 0.03 & 0.04 & 0.02 & 0.14 \\
\hline Nonanoic acid & 34.12 & MS, LRI & 0.01 & 0.05 & 0.01 & nd & nd & nd & nd & 0.14 & 0.05 & 0.09 \\
\hline Benzoic acid & 37.83 & MS, LRI & 0.02 & 0.02 & 0.01 & 0.03 & 0.03 & 0.03 & 0.01 & 0.09 & 0.04 & 0.08 \\
\hline Total & & & 31.76 & 42.91 & 26.20 & 15.80 & 18.55 & 21.46 & 29.24 & 42.67 & 43.43 & 45.93 \\
\hline \multicolumn{13}{|l|}{ Esters } \\
\hline Ethyl acetate & 3.04 & MS, LRI & 0.04 & 0.06 & 0.04 & 0.16 & 0.18 & 0.17 & 0.52 & 0.50 & 0.53 & nd \\
\hline Butanoic acid 3-methyl-ethyl ester & 8.60 & MS, LRI & nd & nd & 0.01 & nd & nd & nd & nd & nd & nd & nd \\
\hline Ethyl lactate & 19.65 & MS, LRI & 0.03 & 0.04 & 0.02 & 0.01 & 0.01 & 0.01 & 0.02 & nd & 0.03 & nd \\
\hline Vinyl 2,2-dimethyl pentanoate & 21.70 & MS, LRI & nd & nd & nd & nd & nd & nd & nd & nd & nd & 0.13 \\
\hline Propylene carbonate & 30.14 & MS, LRI & 0.01 & nd & 0.01 & nd & nd & nd & nd & nd & nd & nd \\
\hline Total & & & 0.08 & 0.10 & 0.08 & 0.17 & 0.20 & 0.18 & 0.54 & 0.50 & 0.56 & 0.13 \\
\hline
\end{tabular}


TABLE 4: Continued.

\begin{tabular}{|c|c|c|c|c|c|c|c|c|c|c|c|c|}
\hline \multirow{2}{*}{ Compounds } & \multirow{2}{*}{$\mathrm{RT}^{\mathrm{a}}$} & \multirow{2}{*}{ Identification $^{\mathrm{b}}$} & \multicolumn{10}{|c|}{ Sample code } \\
\hline & & & $B 1^{\mathrm{c}}$ & $B 2^{\mathrm{c}}$ & $B 3^{\mathrm{c}}$ & $\mathrm{B} 4^{\mathrm{c}}$ & $\mathrm{B} 5^{\mathrm{c}}$ & $B 6^{c}$ & $B 7^{\mathrm{d}}$ & $B 8^{\mathrm{d}}$ & $B 9^{d}$ & $\mathrm{~B} 10^{\mathrm{d}}$ \\
\hline \multicolumn{13}{|l|}{ Aldehydes } \\
\hline Acetaldehyde & 1.75 & MS, LRI & 0.10 & 0.13 & 0.08 & 0.71 & 0.84 & 0.96 & 0.09 & 0.39 & 0.17 & 0.54 \\
\hline Butanal & 2.86 & MS, LRI & 0.75 & 1.01 & 0.62 & 0.02 & 0.05 & 0.08 & 0.61 & 0.20 & 0.53 & 0.02 \\
\hline 2-Methylbutanal & 3.37 & MS, LRI & 1.03 & 1.39 & 0.85 & 0.57 & 0.67 & 0.77 & 1.61 & 0.01 & 0.59 & 0.02 \\
\hline 3-Methylbutanal & 3.45 & MS, LRI & 6.70 & 9.05 & 5.53 & 3.29 & 3.86 & 4.44 & 6.76 & 4.42 & 2.46 & nd \\
\hline Pentanal & 4.79 & MS, LRI & 0.16 & 0.22 & 0.13 & 0.05 & 0.05 & 0.09 & 0.20 & 0.70 & 0.84 & 0.1 \\
\hline Hexanal & 9.01 & MS, LRI & nd & nd & nd & nd & nd & nd & nd & nd & nd & 0.03 \\
\hline 2-Pentanal & 11.54 & MS, LRI & nd & nd & nd & 0.08 & 0.09 & 0.08 & nd & nd & nd & nd \\
\hline Heptanal & 14.09 & MS, LRI & nd & nd & nd & nd & nd & nd & 0.07 & nd & nd & 0.05 \\
\hline Nonanal & 21.00 & MS, LRI & 0.01 & 0.01 & nd & 0.02 & 0.01 & 0.02 & 0.02 & 0.17 & 0.07 & 0.49 \\
\hline Benzaldehyde & 23.89 & MS, LRI & 0.21 & 0.29 & 0.18 & 0.21 & 0.24 & 0.28 & 0.21 & 0.60 & 0.29 & 4.77 \\
\hline Lilac aldehyde A & 24.88 & MS, LRI & nd & nd & nd & nd & nd & nd & nd & nd & nd & nd \\
\hline Lilac aldehyde B & 25.37 & MS, LRI & nd & nd & nd & 0.02 & 0.03 & 0.03 & nd & 0.04 & 0.05 & nd \\
\hline Benzeneacetaldehyde & 26.42 & MS, LRI & 0.61 & 0.82 & 0.50 & 0.06 & 0.07 & 0.08 & 0.06 & nd & 0.08 & 0.09 \\
\hline Nicotinaldehyde & 27.61 & MS, LRI & nd & nd & nd & 0.05 & 0.05 & 0.05 & 0.03 & nd & nd & 0.44 \\
\hline 2-Methyl-2-octenedial & 29.18 & MS, LRI & nd & nd & nd & 0.03 & 0.06 & 0.07 & 0.05 & nd & nd & 0.15 \\
\hline Phenylacetaldehyde & 31.31 & MS, LRI & nd & nd & nd & nd & nd & nd & 0.05 & nd & nd & 0.05 \\
\hline 2-Pyrrolecarboxaldehyde & 32.69 & MS, LRI & 0.18 & 0.25 & 0.15 & 0.30 & 0.35 & 0.33 & 0.71 & 0.12 & 0.10 & nd \\
\hline Cinnamaldehyde & 32.83 & MS, LRI & nd & nd & nd & nd & nd & nd & nd & nd & nd & nd \\
\hline Total & & & 9.75 & 13.16 & 8.04 & 5.41 & 6.37 & 7.30 & 10.48 & 6.65 & 5.18 & 6.63 \\
\hline \multicolumn{13}{|l|}{ Furans } \\
\hline Furan & 2.19 & MS, LRI & nd & nd & nd & nd & nd & nd & nd & nd & nd & 0.68 \\
\hline Methylfuran & 3.15 & MS, LRI & nd & nd & nd & nd & nd & nd & 0.28 & nd & nd & 1.19 \\
\hline Dihydro-2-methyl-3(2H)-furanone & 17.04 & MS, LRI & 0.01 & 0.02 & 0.01 & 0.03 & 0.03 & 0.04 & 0.59 & 0.01 & 0.10 & 0.01 \\
\hline Furfural & 22.61 & MS, LRI & 3.29 & 4.09 & 3.39 & 1.22 & 1.50 & 0.14 & 4.91 & 1.29 & 5.90 & 0.96 \\
\hline 1-(2-Furanyl)-ethanone & 23.54 & MS, LRI & 0.48 & 0.64 & 0.39 & 0.68 & 0.80 & 0.92 & 0.79 & 0.08 & 0.42 & nd \\
\hline 5-Methylfurfural & 25.03 & MS, LRI & 0.04 & 0.04 & 0.04 & 0.16 & 0.19 & 0.22 & 1.25 & 0.50 & 0.52 & 0.05 \\
\hline Dihydro-5-methyl-2(3H)-furanone & 25.65 & MS, LRI & nd & nd & nd & nd & nd & nd & 0.03 & 0.07 & 4.21 & nd \\
\hline Dihydro-3-methyl-2(3H)-furanone & 25.72 & MS, LRI & nd & nd & nd & 1.75 & 2.06 & 1.80 & 1.31 & nd & nd & 0.68 \\
\hline 2(5H)-Furanone & 28.49 & MS, LRI & 0.30 & 0.40 & 0.24 & 0.28 & 0.33 & 0.38 & 0.84 & 0.02 & 0.29 & 0.03 \\
\hline 4,5-Dimethyl-2-furaldehyde & 32.19 & MS, LRI & 0.05 & 0.06 & 0.04 & 0.09 & 0.11 & 0.13 & 0.15 & 0.04 & 0.03 & nd \\
\hline Total & & & 3.69 & 5.25 & 3.72 & 2.31 & 4.23 & 2.70 & 9.37 & 1.93 & 11.05 & 3.60 \\
\hline \multicolumn{13}{|l|}{ Terpenes } \\
\hline Verbenene & 10.74 & MS, LRI & nd & nd & nd & nd & nd & nd & nd & nd & nd & 0.03 \\
\hline$\alpha$-Phellandrene & 12.91 & MS, LRI & nd & nd & nd & nd & nd & nd & nd & nd & nd & 0.03 \\
\hline$\alpha$-Terpinene & 13.62 & MS, LRI & nd & nd & nd & nd & nd & nd & nd & 0.04 & 0.01 & 0.07 \\
\hline$\tau$-Terpinene & 16.45 & MS, LRI & nd & nd & nd & 0.02 & 0.02 & 0.01 & 0.03 & nd & nd & 0.07 \\
\hline Cymene & 17.24 & MS, LRI & nd & nd & nd & nd & nd & nd & 0.65 & nd & nd & 0.08 \\
\hline cis-Linalool oxide & 22.24 & MS, LRI & 0.67 & 0.90 & 0.55 & 4.4 & 5.18 & 5.95 & 3.31 & 6.80 & 11.50 & 2.15 \\
\hline trans-linalool oxide & 22.87 & MS, LRI & 0.46 & 0.62 & 0.38 & 1.23 & nd & 1.66 & 3.23 & 0.28 & 0.25 & nd \\
\hline Menthofuran & 23.06 & MS, LRI & nd & nd & nd & nd & nd & nd & nd & nd & nd & 0.25 \\
\hline Linalool & 24.70 & MS, LRI & 0.13 & 0.17 & 0.11 & 0.11 & 0.13 & 0.15 & 0.13 & 0.20 & 0.29 & 0.35 \\
\hline Damascenone & 29.96 & MS, LRI & nd & nd & nd & 0.01 & 0.28 & 0.02 & 0.09 & nd & nd & nd \\
\hline$\alpha$-Terpinolene & 31.77 & MS, LRI & 0.01 & 0.01 & 0.01 & nd & nd & nd & nd & nd & nd & 0.24 \\
\hline Carvacrol & 34.66 & MS, LRI & nd & 0.06 & nd & nd & nd & nd & nd & nd & nd & 0.09 \\
\hline Total & & & 1.26 & 1.77 & 1.04 & 5.77 & 5.61 & 7.78 & 7.43 & 7.32 & 12.05 & 3.36 \\
\hline \multicolumn{13}{|l|}{ Miscellaneous } \\
\hline Dimethyl sulfide & 1.93 & MS, LRI & 1.94 & 2.61 & 1.60 & 0.76 & 0.89 & 0.83 & 0.90 & 0.62 & 1.16 & 0.92 \\
\hline Eugenol & 34.45 & MS, LRI & 0.01 & nd & nd & nd & nd & nd & nd & nd & nd & nd \\
\hline Total & & & 1.95 & 2.61 & 1.60 & 0.76 & 0.89 & 0.83 & 0.90 & 0.62 & 1.16 & 0.92 \\
\hline
\end{tabular}

${ }^{\mathrm{a}}$ Retention time; ${ }^{\mathrm{b}}$ MS: mass spectrum tentatively identified using NIST 05 and Wiley 275 libraries; LRI: linear retention index.

${ }^{c}$ Normalized amount of volatile compounds (percentage) (peak of volatile compound/total peak area of all volatile compounds) of buckwheat honeys from Italy (Valtellina).

${ }^{\mathrm{d}}$ Normalized amount of volatile compounds (percentage) (peak of volatile compound/total peak area of all volatile compounds) of buckwheat honeys from Russia, Poland, and Nepal.

nd: not detected. 
TABLE 5: Volatile compounds identified in investigated honey samples expressed as percentages. M1-M3: multifloral honeys from Italy (TeglioValtellina); M4-M6: multifloral honeys from Italy (Valtellina); A1-A3: acacia honeys from Italy (Valtellina); R1-R3: rhododendron honeys from Italy (Valtellina).

\begin{tabular}{|c|c|c|c|c|c|c|c|c|c|c|c|c|c|c|}
\hline \multirow{2}{*}{ Compounds } & \multirow{2}{*}{$\mathrm{RT}^{\mathrm{a}}$} & \multirow{2}{*}{ Identification $^{\mathrm{b}}$} & \multicolumn{12}{|c|}{ Sample code } \\
\hline & & & $\mathrm{M1}^{\mathrm{c}}$ & $\mathrm{M} 2^{\mathrm{c}}$ & $\mathrm{M}^{\mathrm{c}}$ & $\mathrm{M} 4^{\mathrm{c}}$ & $M 5^{\mathrm{c}}$ & $M 6^{c}$ & $\mathrm{Al}^{\mathrm{c}}$ & $\mathrm{A} 2^{\mathrm{c}}$ & $\mathrm{A} 3^{\mathrm{c}}$ & $\mathrm{R}^{\mathrm{c}}$ & $\mathrm{R} 2^{\mathrm{c}}$ & $\mathrm{R} 3^{\mathrm{c}}$ \\
\hline \multicolumn{15}{|l|}{ Alcohols } \\
\hline Ethanol & 3.97 & MS, LRI & 25.83 & 15.01 & 6.66 & 30.82 & 28.20 & 34.48 & 13.11 & 15.69 & 9.54 & 40.55 & 32.60 & 38.42 \\
\hline 2,3-Butanedione & 4.98 & MS, LRI & 2.97 & nd & 0.21 & nd & 0.78 & 0.39 & 0.48 & 0.27 & 0.60 & 0.25 & 0.30 & 0.22 \\
\hline 2-Methyl-3-buten-2-ol & 7.39 & MS, LRI & 0.03 & 0.05 & nd & nd & 0.10 & 0.05 & 0.04 & nd & 0.07 & 0.07 & 0.06 & 0.08 \\
\hline 1-Butanol & 12.67 & MS, LRI & 0.08 & 0.06 & 0.08 & 0.5 & 0.68 & 0.41 & 0.45 & 0.11 & 0.78 & 0.38 & 0.25 & 0.68 \\
\hline 2-Methylbutanol & 14.46 & MS, LRI & 0.05 & 0.02 & 0.04 & 0.01 & 0.03 & 0.02 & 0.04 & 0.02 & 0.06 & 0.03 & 0.02 & 0.05 \\
\hline 2-Methylpropanol & 15.02 & MS, LRI & nd & nd & 0.05 & nd & nd & nd & 0.02 & 0.02 & 0.01 & nd & nd & nd \\
\hline 3-Methylbutanol & 15.41 & MS, LRI & 0.58 & 0.47 & 0.56 & 0.39 & 0.51 & 0.55 & 0.54 & 0.25 & 0.92 & 0.51 & 0.35 & 0.60 \\
\hline 2-Methyl-2-buten-1-ol & 16.78 & MS, LRI & 0.14 & 0.06 & 0.10 & 0.01 & 0.10 & 0.09 & 0.10 & 0.07 & 0.14 & 0.12 & 0.08 & 0.10 \\
\hline 3-Methyl-2-buten-1-ol & 16.94 & MS, LRI & nd & nd & nd & nd & nd & nd & nd & nd & nd & nd & nd & nd \\
\hline 2-Methylbutenol & 19.09 & MS, LRI & 0.02 & 0.01 & 0.04 & 0.01 & 0.02 & nd & 0.02 & 0.03 & 0.02 & 0.01 & nd & 0.01 \\
\hline Total & & & 29.70 & 15.68 & 7.74 & 31.73 & 30.42 & 35.99 & 14.80 & 16.46 & 12.14 & 41.92 & 33.66 & 40.16 \\
\hline \multicolumn{15}{|c|}{ 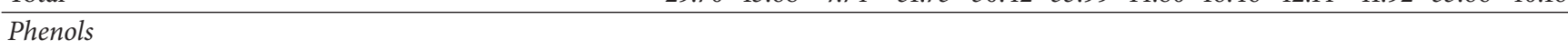 } \\
\hline 2-Caren-10-al & 29.28 & MS, LRI & 0.09 & nd & 0.19 & 0.10 & nd & nd & nd & nd & nd & nd & nd & nd \\
\hline p-Cymen-8-ol & 30.46 & MS, LRI & 0.06 & 0.05 & 0.08 & 0.05 & 0.10 & 0.07 & nd & nd & nd & 0.03 & 0.03 & 0.02 \\
\hline Phenol & 32.53 & MS, LRI & 0.04 & 0.03 & 0.04 & 0.01 & 0.02 & 0.01 & 0.01 & nd & 0.02 & 0.02 & 0.05 & 0.01 \\
\hline p-Cresol & 33.26 & MS, LRI & 0.01 & 0.01 & nd & nd & 0.01 & 0.01 & 0.01 & 0.01 & 0.01 & nd & nd & 0.01 \\
\hline Thymol & 34.34 & & nd & 0.01 & nd & nd & 0.02 & 0.01 & 0.01 & 0.01 & 0.01 & 0.02 & 0.03 & nd \\
\hline Total & & & 0.20 & 0.10 & 0.31 & 0.16 & 0.15 & 0.10 & 0.03 & 0.02 & 0.043 & 0.07 & 0.11 & 0.04 \\
\hline \multicolumn{15}{|l|}{ Ketones } \\
\hline 2-Butanone & 6.61 & MS, LRI & nd & nd & nd & nd & nd & nd & nd & nd & nd & nd & nd & nd \\
\hline 3-Hydroxy-2-butanone & 17.65 & MS, LRI & 1.04 & 1.47 & 0.62 & 1.44 & 0.44 & 0.84 & 1.10 & 1.72 & 1.50 & 0.47 & 0.49 & 0.44 \\
\hline Hydroxyac & 18.08 & MS, LRI & 1.02 & 0.72 & 1.62 & 1.16 & 0.71 & nd & 2.92 & 1.27 & 2.57 & 1.65 & 2.55 & 1.56 \\
\hline 2-Hydroxy-3-pentanone & 19.90 & MS, LRI & nd & nd & nd & nd & nd & nd & nd & nd & nd & nd & nd & nd \\
\hline 1-Hydroxy-2-butanone & 20.29 & MS, LRI & 0.04 & 0.07 & nd & 0.01 & 0.10 & 0.05 & 0.05 & 0.02 & 0.10 & 0.11 & 0.08 & 0.10 \\
\hline 2-Methyl-butyrolactone & 25.20 & MS, LRI & nd & 0.05 & nd & 0.15 & 0.27 & 0.21 & 0.16 & 0.14 & 0.13 & 0.06 & 0.05 & 0.04 \\
\hline 4-Oxoisophorone & 27.42 & MS, LRI & 0.16 & 0.07 & 0.06 & 0.04 & 0.01 & nd & 0.02 & 0.01 & 0.01 & 0.06 & 0.01 & nd \\
\hline Total & & & 2.36 & 2.49 & 2.35 & 2.87 & 1.68 & 1.21 & 4.40 & 3.26 & 4.51 & 3.29 & 3.55 & 2.48 \\
\hline \multicolumn{15}{|l|}{ Free fatty acid } \\
\hline Acetic acid & 22.14 & MS, LRI & 27.4 & 31.32 & 30.48 & 18.50 & 40.64 & 32.56 & 48.12 & 41.40 & 44.83 & 25.63 & 21.53 & 29.74 \\
\hline Formic acid & 23.36 & MS, LRI & 2.58 & 3.13 & 3.03 & 12.08 & 5.49 & 6.29 & 8.29 & 5.56 & 5.03 & 7.53 & 12.25 & 6.82 \\
\hline Propanoic acid & 24.28 & MS, LRI & 0.72 & 0.75 & 0.79 & 0.16 & 0.41 & 0.78 & 0.71 & 0.43 & 0.99 & 0.40 & 0.41 & 0.40 \\
\hline Isobutyric acid & 24.95 & MS, LRI & 1.00 & 1.20 & 1.80 & 1.22 & 2.30 & 1.26 & 1.30 & 0.49 & 1.11 & 0.49 & 0.32 & 0.35 \\
\hline Butanoic acid & 26.19 & MS, LRI & 1.30 & 1.26 & 1.34 & 0.43 & 0.46 & 0.94 & 1.22 & 1.30 & 1.20 & 1.20 & 1.50 & 1.90 \\
\hline 2-Propanoic acid & 26.28 & MS, LRI & 0.05 & 0.02 & 0.08 & nd & 0.01 & nd & 0.05 & 0.02 & 0.08 & 0.01 & 0.01 & nd \\
\hline Isovaleric acid & 27.05 & MS, LRI & 2.73 & 1.70 & 3.74 & 1.41 & 1.47 & 2.94 & 3.50 & 3.17 & 3.82 & 1.71 & 1.43 & 1.99 \\
\hline Pentanoic acid & 28.35 & MS, LRI & 0.43 & 0.03 & 0.23 & 0.07 & 0.18 & 0.18 & 0.24 & 0.25 & 0.28 & nd & 0.02 & nd \\
\hline 2-Butenoic acid & 28.95 & MS, LRI & 0.02 & 0.03 & 0.02 & nd & 0.01 & nd & nd & nd & nd & 0.02 & 0.01 & 0.03 \\
\hline 3-Methylpentanoic acid & 29.40 & MS, LRI & 0.04 & 0.02 & 0.04 & 0.01 & 0.03 & 0.07 & 0.10 & 0.08 & 0.10 & 0.11 & 0.02 & 0.10 \\
\hline 4-Methylpentanoic acid & 29.58 & MS, LRI & nd & nd & nd & nd & nd & nd & nd & nd & nd & nd & nd & nd \\
\hline 4-Pentanoic acid & 30.84 & MS, LRI & nd & nd & nd & nd & nd & nd & nd & nd & nd & nd & nd & nd \\
\hline Benzyl nitrile & 31.63 & MS, LRI & nd & 0.02 & nd & nd & nd & 0.01 & nd & nd & nd & 0.02 & 0.03 & nd \\
\hline Heptanoic acid & 31.96 & MS, LRI & 0.10 & 0.08 & 0.10 & 0.02 & 0.05 & 0.04 & 0.10 & 0.11 & 0.18 & 0.12 & 0.11 & 0.14 \\
\hline Octanoic acid & 33.05 & MS, LRI & 0.07 & 0.03 & 0.03 & nd & 0.01 & 0.01 & 0.03 & 0.04 & 0.04 & 0.01 & 0.02 & nd \\
\hline Nonanoic acid & 34.12 & MS, LRI & 0.03 & 0.02 & 0.03 & nd & 0.02 & nd & 0.02 & nd & 0.02 & 0.01 & 0.01 & nd \\
\hline Benzoic acid & 37.83 & MS, LRI & 0.07 & 0.08 & 0.07 & 0.03 & 0.05 & 0.04 & 0.07 & 0.07 & 0.07 & 0.08 & 0.08 & 0.08 \\
\hline Total & & & 36.54 & 39.69 & 41.78 & 33.93 & 51.13 & 45.12 & 63.75 & 53.37 & 57.76 & 37.34 & 37.75 & 41.55 \\
\hline \multicolumn{15}{|l|}{ Esters } \\
\hline Ethyl acetate & 3.04 & MS, LRI & nd & nd & nd & nd & nd & nd & nd & nd & nd & 0.04 & 0.08 & 0.05 \\
\hline Butanoic acid 3-methyl-ethyl ester & 8.60 & MS, LRI & nd & 0.02 & 0.01 & 0.05 & nd & 0.03 & nd & nd & 0.03 & nd & nd & nd \\
\hline Ethyl lactate & 19.65 & MS, LRI & 0.03 & 0.04 & nd & 0.01 & nd & 0.01 & nd & 0.02 & nd & 0.02 & nd & 0.04 \\
\hline Vinyl 2,2-dimethyl pentanoate & 21.70 & MS, LRI & 0.13 & 0.10 & 0.25 & nd & 0.1 & nd & nd & nd & nd & nd & nd & nd \\
\hline Propylene carbonate & 30.14 & MS, LRI & nd & nd & nd & nd & nd & nd & nd & nd & nd & 0.01 & 0.02 & 0.01 \\
\hline Total & & & 0.16 & 0.16 & 0.26 & 0.06 & 0.10 & 0.04 & nd & 0.02 & 0.03 & 0.07 & 0.10 & 0.10 \\
\hline
\end{tabular}


TABLE 5: Continued.

\begin{tabular}{|c|c|c|c|c|c|c|c|c|c|c|c|c|c|c|}
\hline \multirow{2}{*}{ Compounds } & \multirow{2}{*}{$\mathrm{RT}^{\mathrm{a}}$} & \multirow{2}{*}{ Identification $^{\mathrm{b}}$} & \multicolumn{12}{|c|}{ Sample code } \\
\hline & & & $\mathrm{M1}^{\mathrm{c}}$ & $\mathrm{M} 2^{\mathrm{c}}$ & $\mathrm{M}^{\mathrm{c}}$ & $\mathrm{M} 4^{\mathrm{c}}$ & $\mathrm{M} 5^{\mathrm{c}}$ & $M 6^{\mathrm{c}}$ & $\mathrm{Al}^{\mathrm{c}}$ & $\mathrm{A} 2^{\mathrm{c}}$ & $\mathrm{A} 3^{\mathrm{c}}$ & $\mathrm{R}^{\mathrm{c}}$ & $\mathrm{R} 2^{\mathrm{c}}$ & $\mathrm{R} 3^{\mathrm{c}}$ \\
\hline \multicolumn{15}{|l|}{ Aldehydes } \\
\hline Acetaldehyde & 1.75 & MS, LRI & 0.46 & 0.52 & 0.40 & 0.91 & 0.41 & 0.45 & nd & nd & nd & 0.37 & 0.20 & 0.46 \\
\hline Butanal & 2.86 & MS, LRI & nd & nd & nd & nd & nd & nd & nd & nd & nd & nd & nd & nd \\
\hline 2-Methylbutanal & 3.37 & MS, LRI & nd & nd & nd & nd & nd & nd & nd & nd & nd & nd & nd & nd \\
\hline 3-Methylbutanal & 3.45 & MS, LRI & nd & nd & nd & nd & nd & nd & nd & nd & nd & 0.05 & 0.10 & 0.05 \\
\hline Pentanal & 4.79 & MS, LRI & nd & nd & nd & nd & nd & nd & nd & nd & nd & nd & nd & nd \\
\hline Hexanal & 9.01 & MS, LRI & nd & nd & nd & nd & nd & nd & 0.04 & nd & 0.07 & nd & nd & 0.01 \\
\hline 2-Pentanal & 11.54 & MS, LRI & nd & nd & nd & nd & nd & nd & 0.01 & 0.01 & nd & nd & nd & nd \\
\hline Heptanal & 14.09 & MS, LRI & 0.03 & 0.01 & 0.02 & nd & 0.03 & 0.02 & nd & nd & nd & nd & nd & nd \\
\hline Nonanal & 21.00 & MS, LRI & 0.06 & 0.02 & 0.05 & 0.05 & nd & nd & 0.08 & 0.15 & nd & nd & nd & nd \\
\hline Benzaldehyde & 23.89 & MS, LRI & 1.19 & 1.06 & 0.70 & 0.40 & 0.96 & 0.53 & 0.86 & 0.60 & 1.11 & 1.70 & 1.10 & 1.30 \\
\hline Lilac aldehyde A & 24.88 & MS, LRI & 1.00 & 1.20 & 0.80 & 0.22 & 1.30 & 1.26 & 0.02 & 0.02 & 0.01 & nd & nd & nd \\
\hline Lilac aldehyde B & 25.37 & MS, LRI & 0.10 & 0.08 & nd & 0.02 & 0.06 & 0.04 & 0.07 & 0.09 & 0.07 & 0.16 & 0.03 & 0.10 \\
\hline Benzeneacetaldehyde & 26.42 & MS, LRI & nd & 0.01 & nd & 0.01 & 0.02 & 0.04 & 0.05 & 0.04 & 0.07 & 0.06 & 0.03 & 0.04 \\
\hline Nicotinaldehyde & 27.61 & MS, LRI & 0.50 & 0.34 & 0.60 & nd & 0.04 & nd & nd & nd & nd & 0.22 & 0.10 & 0.14 \\
\hline 2-Methyl-2-octenedial & 29.18 & MS, LRI & 0.10 & 0.03 & 0.10 & 0.01 & 0.10 & 0.11 & nd & nd & nd & 0.02 & 0.03 & 0.01 \\
\hline Phenylacetaldehyde & 31.31 & MS, LRI & 0.03 & nd & 0.07 & nd & nd & nd & nd & 0.02 & nd & nd & nd & nd \\
\hline 2-Pyrrolecarbaldehyde & 32.69 & MS, LRI & nd & 0.03 & nd & 0.01 & nd & 0.01 & 0.02 & 0.02 & 0.01 & 0.01 & 0.02 & nd \\
\hline Cinnamaldehyde & 32.83 & MS, LRI & 0.03 & nd & 0.02 & 0.03 & 0.05 & 0.01 & 0.01 & nd & 0.02 & nd & 0.02 & nd \\
\hline Total & & & 3.50 & 3.30 & 2.76 & 1.65 & 2.97 & 2.47 & 1.16 & 0.96 & 1.37 & 2.59 & 1.63 & 2.11 \\
\hline \multicolumn{15}{|l|}{ Furans } \\
\hline Furan & 2.19 & MS, LRI & 0.18 & nd & 0.03 & nd & 0.02 & nd & nd & nd & nd & nd & nd & nd \\
\hline Methylfuran & 3.15 & MS, LRI & 0.81 & 0.27 & 0.30 & 0.25 & 0.15 & nd & nd & nd & nd & 0.13 & 0.10 & 0.25 \\
\hline Dihydro-2-methyl-3(2H)-furanone & 17.04 & MS, LRI & nd & nd & nd & nd & nd & nd & nd & nd & nd & nd & nd & nd \\
\hline Furfural & 22.61 & MS, LRI & 1.42 & 1.71 & 1.13 & 1.07 & 1.30 & 2.19 & 3.41 & 2.06 & 4.77 & 2.56 & 2.53 & 2.50 \\
\hline 1-(2-Furanyl)-ethanone & 23.54 & MS, LRI & 0.05 & 0.10 & nd & 0.05 & 0.10 & 0.08 & 0.10 & 0.05 & 0.15 & 0.5 & 0.08 & 0.01 \\
\hline 5-Methylfurfural & 25.03 & MS, LRI & nd & nd & nd & nd & nd & nd & nd & nd & nd & nd & nd & nd \\
\hline Dihydro-5-methyl-2(3H)-furanone & 25.65 & MS, LRI & 0.75 & nd & 0.09 & 0.01 & 0.05 & 0.03 & 0.05 & 0.05 & 0.05 & nd & nd & 0.01 \\
\hline Dihydro-3-methyl-2(3H)-furanone & 25.72 & MS, LRI & 0.40 & 0.38 & 0.70 & 0.04 & 0.09 & 0.07 & nd & nd & nd & 0.44 & 0.87 & 0.50 \\
\hline 2(5H)-Furanone & 28.49 & MS, LRI & nd & 0.03 & nd & nd & 0.04 & 0.02 & nd & nd & 0.02 & 0.01 & 0.02 & nd \\
\hline 4,5-Dimethyl-2-furaldehyde & 32.19 & MS, LRI & nd & 0.02 & nd & 0.02 & 0.03 & 0.02 & 0.04 & 0.02 & 0.05 & 0.07 & 0.08 & 0.05 \\
\hline Total & & & 3.56 & 2.41 & 2.25 & 1.39 & 1.68 & 2.33 & 3.50 & 2.13 & 4.89 & 3.25 & 3.60 & 3.31 \\
\hline \multicolumn{15}{|l|}{ Terpenes } \\
\hline Verbenene & 10.74 & MS, LRI & nd & nd & nd & nd & 0.03 & 0.01 & nd & nd & nd & nd & nd & nd \\
\hline$\alpha$-Phellandrene & 12.91 & MS, LRI & 0.03 & nd & 0.05 & nd & 0.03 & nd & nd & nd & nd & nd & nd & nd \\
\hline$\alpha$-Terpinene & 13.62 & MS, LRI & 0.06 & 0.01 & 0.03 & 0.04 & 0.02 & nd & nd & 0.05 & 0.07 & nd & nd & nd \\
\hline$\tau$-Terpinene & 16.45 & MS, LRI & nd & nd & nd & nd & nd & nd & nd & 0.01 & nd & nd & nd & nd \\
\hline Cymene & 17.24 & MS, LRI & 0.05 & 0.01 & 0.05 & nd & 0.01 & nd & nd & nd & nd & nd & nd & nd \\
\hline cis-Linalool oxide & 22.24 & MS, LRI & nd & nd & nd & nd & nd & nd & nd & nd & nd & nd & nd & nd \\
\hline trans-Linalool oxide & 22.87 & MS, LRI & 0.19 & 0.10 & 0.25 & 0.03 & nd & 0.01 & 0.11 & 0.08 & 0.04 & 0.14 & 0.06 & 0.10 \\
\hline Menthofuran & 23.06 & MS, LRI & 0.26 & nd & 0.50 & nd & 0.10 & nd & nd & nd & nd & nd & nd & nd \\
\hline Linalool & 24.70 & MS, LRI & 0.75 & 0.20 & 0.23 & 0.12 & 0.18 & nd & 0.47 & 0.65 & 0.20 & 0.91 & 0.12 & 0.67 \\
\hline Damascenone & 29.96 & MS, LRI & 0.02 & 0.02 & nd & 0.01 & nd & 0.01 & nd & 0.02 & nd & 0.13 & 0.10 & 0.12 \\
\hline$\alpha$-Terpinolene & 31.77 & MS, LRI & 0.14 & nd & 0.25 & nd & 0.11 & 0.05 & nd & nd & 0.03 & 0.01 & 0.02 & nd \\
\hline Carvacrol & 34.66 & MS, LRI & nd & nd & 0.01 & nd & 0.07 & 0.03 & nd & nd & nd & nd & nd & nd \\
\hline Total & & & 1.5 & 0.34 & 1.37 & 0.20 & 0.55 & 0.11 & 0.58 & 0.00 & 0.34 & 1.19 & 0.30 & 0.89 \\
\hline \multicolumn{15}{|l|}{ Miscellanous } \\
\hline Dimethyl sulfide & 1.93 & MS, LRI & 1.07 & 1.14 & 0.70 & nd & 1.00 & nd & nd & nd & nd & 0.72 & 0.15 & 1.20 \\
\hline Eugenol & 34.45 & MS, LRI & nd & 0.01 & nd & nd & 0.01 & 0.01 & 0.01 & 0.02 & 0.01 & nd & nd & nd \\
\hline Total & & & 1.07 & 1.15 & 0.70 & nd & 1.01 & 0.01 & 0.01 & 0.02 & 0.01 & 0.72 & 0.15 & 1.20 \\
\hline
\end{tabular}

${ }^{\mathrm{a}}$ Retention time; ${ }^{\mathrm{b}}$ MS: mass spectrum tentatively identified using NIST 05 and Wiley 275 libraries; LRI: linear retention index.

${ }^{c}$ Normalized amount of volatile compounds (percentage) (peak of volatile compound/total peak area of all volatile compounds) of multifloral, acacia, and rhododendron honey from Italy (Valtellina).

nd: not detected. 
Poland, classified as multifloral honey but containing a significant level of buckwheat pollen grains, were found to have a very similar volatile organic compounds profile, despite the different geographical origin. Thus, the VOCs profile analyses seemed to be useful in distinguishing honeys containing buckwheat pollen grains from those of different botanical origin.

Many volatile compounds were identified in all honey types, but in the honeys containing buckwheat pollen grains there were components that were not present in other honey types such as 3-methyl-2-buten-1-ol, 2-butanone, 2-hydroxy3-pentanone, 4-methylpentanoic acid, 4-pentanoic acid, butanal, 2-methylbutanal, pentanal, dihydro-2-methyl-3(2H)furanone, 5-methylfurfural, and cis-linalool oxide. Among them, butanal and 2-methylbutanal have been proposed as buckwheat honey markers also by other authors [22, 32, 33].

According to the literature, butanoic acid and pentanoic acid were considerably greater in the buckwheat honey samples particularly in those from Italy and Poland containing the higher level of buckwheat pollen grains. These compounds have been reported to give buckwheat honey its characteristic aromatic and organoleptic properties and are to be considered interesting as potential "variety markers". Finally, isovaleric acid, whose presence is reported to have a negative sensorial impact, was for the first time detected in buckwheat honey, particularly in the Italian monofloral buckwheat honeys and in the Polish samples.

\section{Conflict of Interests}

The authors declare that they have no conflict of interests.

\section{Acknowledgments}

This study was partly supported by a grant from the PIC Interreg Italia-Svizzera 2007-2013 "Proalpi-valori e sapori delle produzioni tradizionali alpine" and partly by "Accordo di Programma, affermazione in Edolo del Centro di Eccellenza Università della Montagna” MIUR-Università degli Studi di Milano, protocol no. 386 1293-05/08/2011.

\section{References}

[1] M. V. Baroni, M. L. Nores, M. D. P. Díaz et al., "Determination of volatile organic compound patterns characteristic of five unifloral honey by solid-phase microextraction-gas chromatographymass spectrometry coupled to chemometrics," Journal of Agricultural and Food Chemistry, vol. 54, no. 19, pp. 7235-7241, 2006.

[2] E. Alissandrakis, D. Daferera, P. A. Tarantilis, M. Polissiou, and P. C. Harizanis, "Ultrasound-assisted extraction of volatile compounds from citrus flowers and citrus honey," Food Chemistry, vol. 82, no. 4, pp. 575-582, 2003.

[3] G. Aronne and V. de Micco, "Traditional melissopalynology integrated by multivariate analysis and sampling methods to improve botanical and geographical characterisation of honeys," Plant Biosystems, vol. 144, no. 4, pp. 833-840, 2010.

[4] S. Ampuero, S. Bogdanov, and J.-O. Bosset, "Classification of unifloral honeys with an MS-based electronic nose using different sampling modes: SHS, SPME and INDEX," European Food Research and Technology, vol. 218, no. 2, pp. 198-207, 2004.
[5] I. Stanimirova, B. Üstün, T. Cajka et al., "Tracing the geographical origin of honeys based on volatile compounds profiles assessment using pattern recognition techniques," Food Chemistry, vol. 118, no. 1, pp. 171-176, 2010.

[6] C. Guyot, V. Scheirman, and S. Collin, "Floral origin markers of heather honeys: Calluna vulgaris and Erica arborea," Food Chemistry, vol. 64, no. 1, pp. 3-11, 1999.

[7] A. C. Soria, M. González, C. de Lorenzo, I. Martínez-Castro, and J. Sanz, "Characterization of artisanal honeys from Madrid (Central Spain) on the basis of their melissopalynological, physicochemical and volatile composition data," Food Chemistry, vol. 85, no. 1, pp. 121-130, 2004.

[8] V. Kaškoniene and P. R. Venskutonis, "Floral markers in honey of various botanical and geographic origins: a review," Comprehensive Reviews in Food Science and Food Safety, vol. 9, no. 6, pp. 620-634, 2010.

[9] A. Giorgi, M. Madeo, J. Baumgartner, and G. C. Lozzia, "The relationships between phenolic content, pollen diversity, physicochemical information and radical scavenging activity in honey," Molecules, vol. 16, no. 1, pp. 336-347, 2011.

[10] F. Tornuk, S. Karaman, I. Ozturk et al., "Quality characterization of artisanal and retail Turkish blossom honeys: determination of physicochemical, microbiological, bioactive properties and aroma profile," Industrial Crops and Products, vol. 46, pp. 124$131,2013$.

[11] I. Jerković and Z. Marijanović, "Volatile composition screening of Salix spp. nectar honey: benzenecarboxylic acids, norisoprenoids, terpenes, and others," Chemistry and Biodiversity, vol. 7, no. 9, pp. 2309-2325, 2010.

[12] F. Bianchi, M. Careri, and M. Musci, "Volatile norisoprenoids as markers of botanical origin of Sardinian strawberry-tree (Arbutus unedo L.) honey: characterisation of aroma compounds by dynamic headspace extraction and gas chromatography-mass spectrometry," Food Chemistry, vol. 89, no. 4, pp. 527-532, 2005.

[13] J. S. Bonvehí and F. Ventura, "Flavour index and aroma profiles of fresh and processed honeys," Journal of the Science of Food and Agriculture, vol. 83, no. 4, pp. 275-282, 2003.

[14] I. Jerković, Z. Marijanović, J. Kezić, and M. Gugić, "Headspace, volatile and semi-volatile organic compounds diversity and radical scavenging activity of ultrasonic solvent extracts from Amorpha fruticosa honey samples," Molecules, vol. 14, no. 8, pp. 2717-2728, 2009.

[15] W. Wardencki, T. Chmiel, T. Dymerski, P. Biernacka, and B. Plutowska, "Application of gas chromatography, mass spectrometry and olfactometry for quality assessment of selected food products," Ecological Chemistry and Engineering S, vol. 16, pp. 287300, 2009.

[16] T. Björkman, Guide To Buckwheat Production in the Northeast. N.Y. State Agr. and Ext. Services, Cornell University, Geneva, NY, USA, 2002.

[17] D. Janovská, J. Kalinová, and A. Michalová, Metodika pestování pohanky $v$ ekologickém a konvencním zemedelství. Metodika pro praxi, Výzkumný ústav rostlinné výroby, Prague, Czech Republic, 2009.

[18] I. Fontanari, "Un "antico" cereale da riscoprire: il grano saraceno," Terra Trentina, vol. 5, pp. 46-47, 2004.

[19] B. Borghi, C. Minoia, M. Cattaneo et al., "Primi risultati di prove agronomiche e varietali," L'Informatore Agrario, vol. 25, pp. 5963, 1995.

[20] B. Borghi, "Il grano saraceno, coltura che ha futuro," Vita in Campagna, vol. 10, pp. 46-48, 1996. 
[21] F. Pasini, S. Gardini, G. L. Marcazzan, and M. F. Caboni, "Buckwheat honey: screening of composition and properties," Food Chemistry, vol. 141, pp. 2802-2811, 2013.

[22] R. Dalby, "Classic American honey plant: buckwheat," American Bee Journal, vol. 140, pp. 485-486, 2000.

[23] J. Zhou, P. Li, N. Cheng et al., "Protective effects of buckwheat honey on DNA damage induced by hydroxyl radicals," Food and Chemical Toxicology, vol. 50, no. 8, pp. 2766-2773, 2012.

[24] K. Brudzynski, K. Abubaker, and T. Wang, "Powerful bacterial killing by buckwheat honeys is concentration-dependent, involves complete DNA degradation and requires hydrogen peroxide," Frontiers in Microbiology, vol. 3, p. 242, 2012.

[25] C. E. Manyi-Loh, A. M. Clarke, and R. N. Ndip, "An overview of honey: therapeutic properties and contribution in nutrition and human health," African Journal of Microbiology Research, vol. 5, no. 8, pp. 844-852, 2011

[26] J. Louveaux, A. Maurizio, and G. Vorwhol, Method of Mellissopalynology, International Commission for Bee Botany of IUBS, 1970.

[27] G. Ricciardelli D’albore and L. Persano Oddo, Flora Apistica Italiana, Istituto sperimentale per la zoologia agraria, 1978.

[28] O. M. Barth, "Microscopical analysis of honey samples," Anais da Academia Brasileira de Ciências, vol. 42, no. 2, pp. 351-366, 1970 (Portuguese).

[29] S. Panseri, S. Soncin, L. M. Chiesa, and P. A. Biondi, "A headspace solid-phase microextraction gas-chromatographic massspectrometric method (HS-SPME-GC/MS) to quantify hexanal in butter during storage as marker of lipid oxidation," Food Chemistry, vol. 127, no. 2, pp. 886-889, 2011.

[30] S. Soncin, L. M. Chiesa, S. Panseri, P. Biondi, and C. Cantoni, "Determination of volatile compounds of precooked prawn (Penaeus vannamei)and cultured gilthead sea bream (Sparus aurata) stored in ice as possible spoilage markers using solid phase microextraction and gas chromatography/mass spectrometry," Journal of the Science of Food and Agriculture, vol. 89, no. 3, pp. 436-442, 2009.

[31] G. Bentivenga, M. D’Auria, P. Fedeli, G. Mauriello, and R. Racioppi, "SPME-GC-MS analysis of volatile organic compounds in honey from Basilicata. Evidence for the presence of pollutants from anthropogenic activities," International Journal of Food Science and Technology, vol. 39, no. 10, pp. 1079-1086, 2004.

[32] T. Wolski, K. Tambor, H. Rybak-Chmielewska, and B. Kędzia, "Identification of honey volatile components by solid phase microextraction (SPME) and gas chromatography/mass spectrometry (GC/MS)," Journal of Apicultural Science, vol. 50, no. 2, pp. 115-126, 2006.

[33] B. Plutowska, T. Chmiel, T. Dymerski, and W. Wardencki, "A headspace solid-phase microextraction method development and its application in the determination of volatiles in honeys by gas chromatography," Food Chemistry, vol. 126, no. 3, pp. 12881298, 2011.

[34] L. Piasenzotto, L. Gracco, and L. Conte, "Solid phase microextraction (SPME) applied to honey quality control," Journal of the Science of Food and Agriculture, vol. 83, no. 10, pp. 1037-1044, 2003.

[35] E. Alissandrakis, P. A. Tarantilis, P. C. Harizanis, and M. Polissiou, "Evaluation of four isolation techniques for honey aroma compounds," Journal of the Science of Food and Agriculture, vol. 85, no. 1, pp. 91-97, 2005.

[36] E. de la Fuente, I. Martínez-Castro, and J. Sanz, "Characterization of Spanish unifloral honeys by solid phase microextraction and gas chromatography-mass spectrometry," Journal of Separation Science, vol. 28, no. 9-10, pp. 1093-1100, 2005.

[37] Q. Zhou, C. L. Wintersteen, and K. R. Cadwallader, "Identification and quantification of aroma-active components that contribute to the distinct malty flavor of buckwheat honey," Journal of Agricultural and Food Chemistry, vol. 50, no. 7, pp. 2016-2021, 2002.

[38] B. Fickert and P. Schieberle, "Identification of the key odorants in barley malt (caramalt) using GC/MS techniques and odour dilution analyses," Die Nahrung, vol. 42, no. 6, pp. 371-375, 1998.

[39] M. Shimoda, Y. Wu, and Y. Osajima, "Aroma compounds from aqueous solution of haze (Rhus succedanea) honey determined by adsorptive column chromatography," Journal of Agricultural and Food Chemistry, vol. 44, no. 12, pp. 3913-3918, 1996.

[40] R. F. A. Moreira, L. C. Trugo, M. Pietroluongo, and C. A. B. de Maria, "Flavor composition of cashew (Anacardium occidentale) and marmeleiro (Croton species) honeys," Journal of Agricultural and Food Chemistry, vol. 50, no. 26, pp. 7616-7621, 2002.

[41] C. A. B. de Maria and R. F. A. Moreira, "Volatile compounds in floral honeys," Quimica Nova, vol. 26, no. 1, pp. 90-96, 2003. 

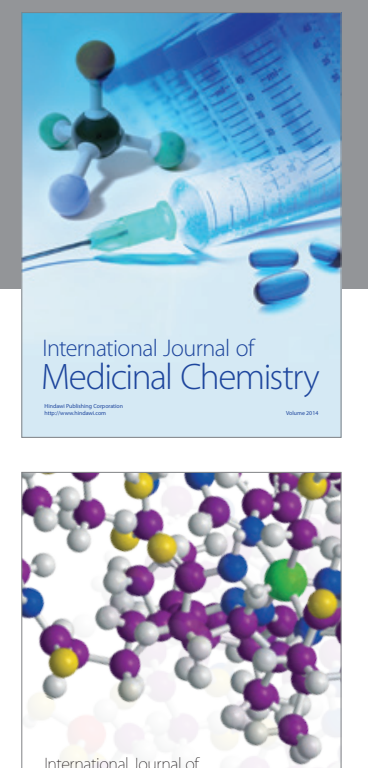

\section{Carbohydrate} Chemistry

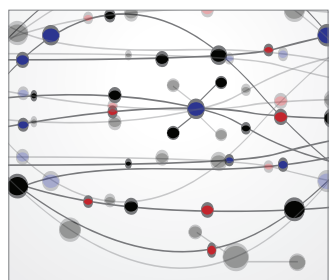

The Scientific World Journal
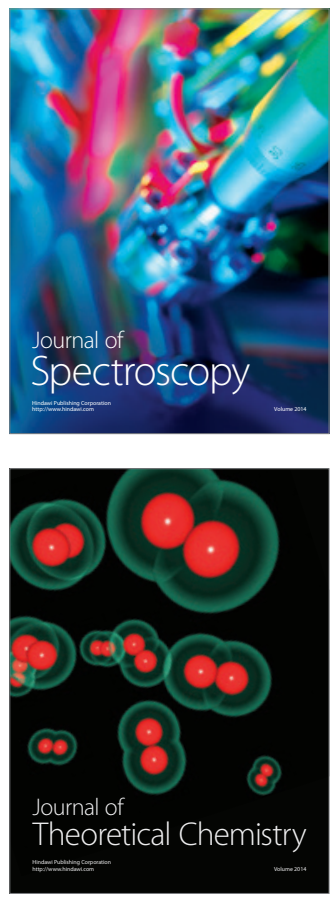
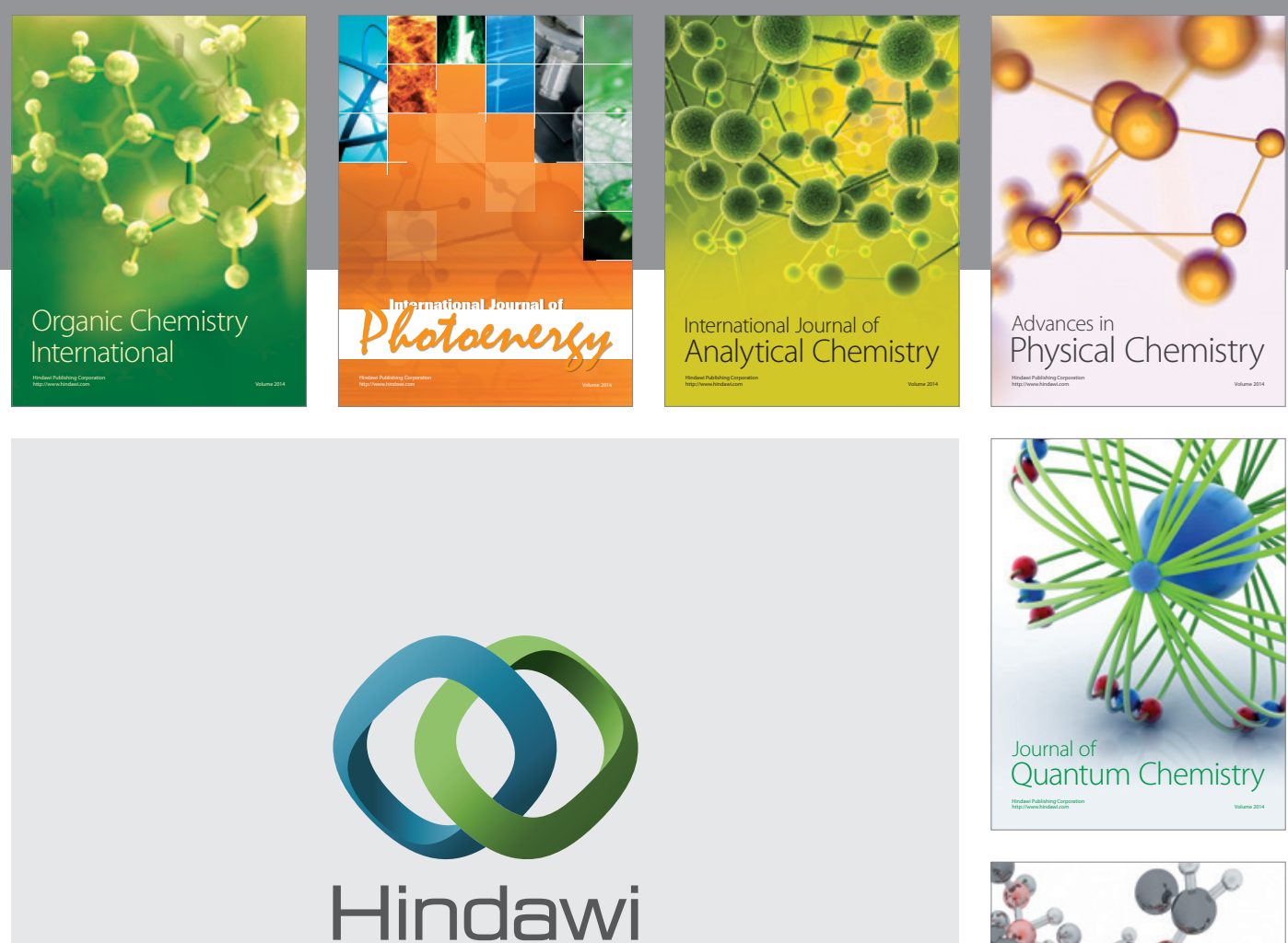

Submit your manuscripts at

http://www.hindawi.com

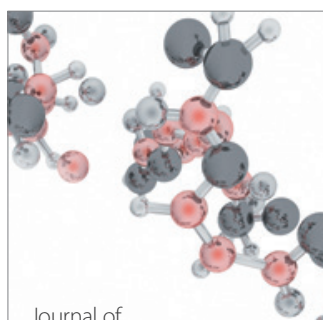

Analytical Methods

in Chemistry

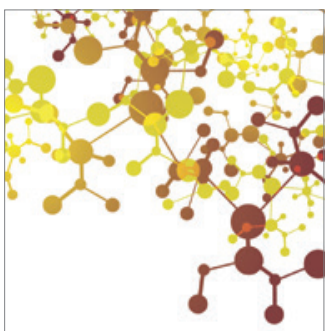

Journal of

Applied Chemistry

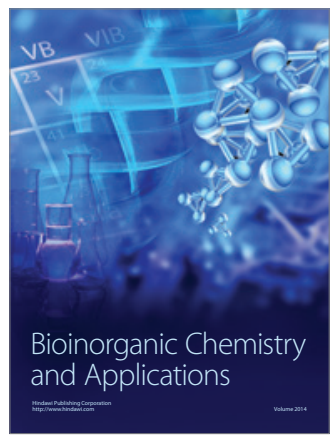

Inorganic Chemistry
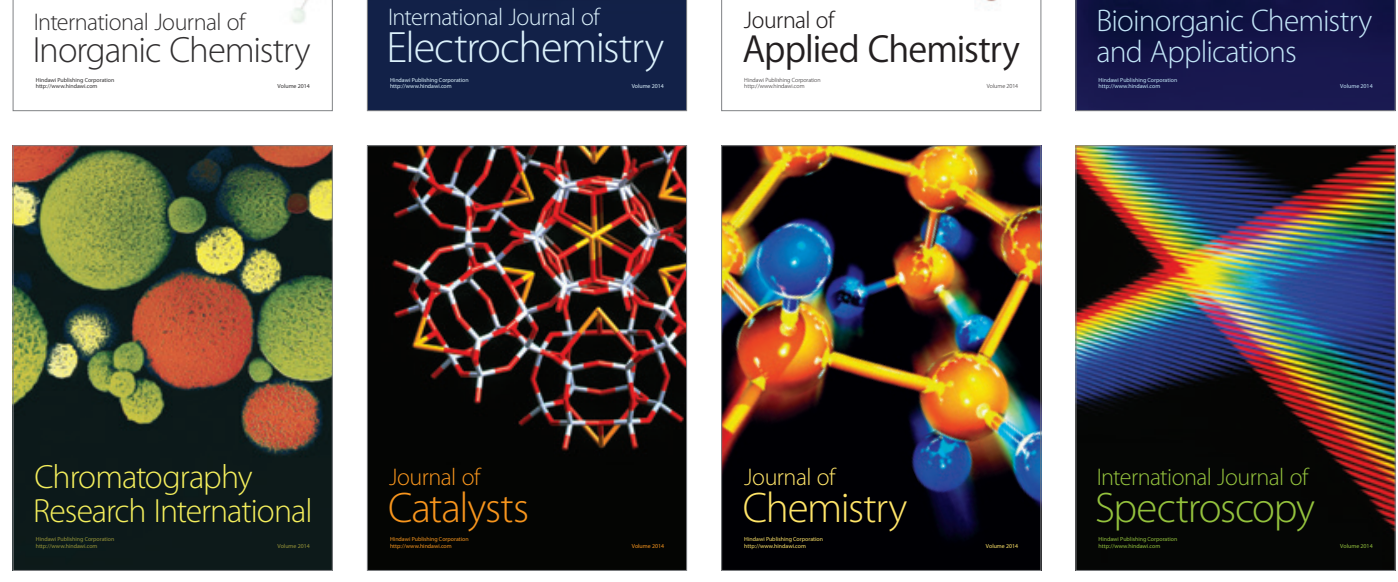